\title{
Treatment of Stenotrophomonas neonatal urinary tract infection with instillation of ciprofloxacin
}

\author{
Mohammad Khassawneh • Wail Hayajneh
}

Received: 26 December 2009/Revised: 8 January 2010/Accepted: 12 January 2010/Published online: 18 February 2010

(C) IPNA 2010

Stenotrophomonas maltophilia is an aerobic Gram-negative bacterium that causes infections that are difficult to treat [1]. It is mostly seen in ill patients exposed to broad spectrum antibiotics and particularly in patients with indwelling catheters or foreign bodies. This bacterium is usually not susceptible to commonly used antibacterial agents [2]. Infection by highly resistant Stenotrophomonas maltophilia is known to cause severe infection, septic shock and meningitis with a high mortality rate, especially in severely ill patients [3]. However, treating these bacteria with effective antibiotics is associated with a better outcome [1]. Although the use of ciprofloxacin in treating Stenotrophomonas maltophilia is frequently reported [3], its use in the neonatal age group is still not universally accepted. Animal studies showed concerns regarding the side effect chondrotoxicity. However, in 1994, Berkovitch et al. reported the use of ciprofloxacin in 35 pregnant women in their first trimester with no apparent abnormalities seen in their newborns [4]. Local instillation of this antibiotic may help avoid taking the risk of administering systemic treatment.

We report a case of urinary tract infection (UTI) caused by Stenotrophomonas maltophilia in a male newborn with congenital urinary tract obstruction and large left-sided urinoma. A nephrostomy tube was placed in the left kidney to decompress the urinoma and to relieve obstruction. This patient developed UTI caused by cotrimoxazole-resistant

M. Khassawneh $(\bowtie) \cdot$ W. Hayajneh

Department of Pediatrics,

Jordan University of Science and Technology,

Irbid, Jordan

e-mail: deema321@yahoo.com
Stenotrophomonas maltophilia on the 47th day of life. Treatment with a full course of antibiotics using cotrimoxazole and imepenem did not clear this infection. The patient partially responded to a full course of treatment with intravenous (IV) ciprofloxacin, but Stenotrophomonas maltophilia grew 3 days after stopping treatment. At that time IV ciprofloxacin was used as well as locally instilled ciprofloxacin in the nephrostomy tube. The dose used in the instillation was $10 \mathrm{mg} / \mathrm{kg}$ (a total of $30 \mathrm{mg}$ ). The $30 \mathrm{mg}$ was then diluted in $60 \mathrm{ml}$ normal saline and divided into six doses. Each dose was instilled locally for 30 min once every $4 \mathrm{~h}$ for 14 days. This completely treated the infection. Patient tolerated this treatment with no apparent local irritation.

In patients in this age group with nephrostoma, an approach using a combination of local and systemic antibiotics to treat UTI was successful. One might speculate whether in non-febrile infants local instillation of ciprofloxacin might be successful to eradicate UTI caused by resistant bacteria.

\section{References}

1. Metan G, Uzun O (2005) Impact of initial antimicrobial therapy in patients with bloodstream infections caused by Stenotrophomonas maltophilia. Antimicrob Agents Chemother 49:3980-3981

2. Nicodemo AC, Paez JI (2007) Antimicrobial therapy for Stenotrophomonas maltophilia infections. Eur J Clin Microbiol Infect Dis 26:229-237

3. Lo WT, Wang CC, Lee CM, Chu ML (2002) Successful treatment of multi-resistant Stenotrophomonas maltophilia meningitis with ciprofloxacin in a pre-term infant. Eur J Pediatr 161:680-682

4. Berkovitch M, Pastuszak A, Gazarian M, Lewis M, Koren G (1994) Safety of new quinolones in pregnancy. Obstet Gynecol 84:535-538 